\title{
LA COMUNIDAD DE SAN RAMÓN DE LA VIRGEN DE SARAPIQUÍ: UN ESTUDIO DE CASO SOBRE TURISMO RURAL Y EMPODERAMIENTO DE MUJERES
}

\author{
Patricia López Estrada ${ }^{1}$ y Lady Fernández Mora² \\ 1 y2 Instituto Tecnológico de Costa Rica, Costa Rica. plopez@itcr.ac.cr; Ifernandez@itcr.ac.cr
}

\begin{abstract}
Resumen. El empoderamiento comunitario juega un papel preponderante en la sociedad. Las comunidades rurales empoderadas son fundamentales para el crecimiento socioeconómico de cualquier región. La literatura indica que el empoderamiento de las mujeres puede ayudar con el desarrollo comunitario (Buendía-Martínez y Carrasco, 2013; Jiménez y López, 2014; Soto y Fawaz, 2016). Este estudio de caso, basado en la investigación cualitativa, tiene como propósito describir las percepciones de cinco mujeres de una comunidad rural después de haber sido capacitadas en turismo rural durante el 2015-2018, para explorar cómo estas percepciones contribuyen con el empoderamiento de su comunidad. Tomando en cuenta la Teoría Feminista (Grbich, 2009), este estudio exploratorio utilizará el análisis de contenido (Hatch, 2002), en su enfoque temático (Braun y Clarke, 2006; Clark y Braun, 2013), para categorizar los temas que representan las perspectivas de las mujeres. Los datos serán recolectados y triangulados a través de entrevistas semiestructuras, observación de campo y recolección de documentación. Este estudio está en curso debido a la crisis de salud mundial del COVID-19. Una vez que se se vuelva a los procesos sociales de una forma cotidiana, los resultados buscarán una comprensión más consolidada de las experiencias y el rol que juegan las mujeres en la comunidad rural.
\end{abstract}

Palabras clave: Comunidad; Empoderamiento; Mujer; Rural; Turismo.

\section{SAN RAMÓN DE LA VIRGEN DE SARAPIQUÍ: A CASE STUDY ABOUT RURAL TOURISM AND WOMEN EMPOWERMENT}

\begin{abstract}
Community empowerment plays a major role in society. Empowering communities in rural areas becomes fundamental for the socioeconomic growth of any region. The literature indicates that the empowerment of women can help increase their community development (Buendía-Martínez \& Carrasco, 2013; Jiménez \& López, 2014; Soto \& Fawaz, 2016). This case study, based on qualitative research, aims at describing the perceptions of five women in a rural community after a training process of rural tourism during 2015-2018, to explore how these perceptions contribute to their community empowerment. Based on Feminist Theory (Grbich, 2009), the exploratory study will use content analysis (Hatch, 2002), in its thematic approach (Braun \& Clarke, 2006; Clark \& Braun, 2013) to categorize themes to present the women's views. Data will be collected and triangulated through semi-structured interviews, field observation, and document gathering. The study will take place once there is a steady social normality due to the COVID-19 world crisis. The results will aim at better comprehending the experiences and the role women play in the rural community.
\end{abstract}

Keywords: Comunity; Empowerment; Rural; Tourism; Women.

\section{INTRODUCCIÓN}

Esta investigación cualitativa se basa en los alcances que tuvieron dos proyectos de extensión universitarios: "Aprendiendo sobre la actividad turística como un medio para complementar las actividades primarias de la comunidad de San Ramón, Sarapiquí (20152016)" y "Fortalecimiento del emprendedurismo de los asociados de Cooproturs R.L. en la 
comunidad de San Ramón de La Virgen, Sarapiquí" (2017-2018). Estos proyectos fueron concebidos y llevados a cabo por la carrera en Gestión del Turismo Rural Sostenible de la Escuela de Idiomas y Ciencias Sociales del Campus Tecnológico Local San Carlos. Los dos proyectos de extensión constituyeron un proceso de empoderamiento de la comunidad rural y promovieron el desarrollo de un papel protagónico de las mujeres en la comunidad. La Cooperativa Agroecoturística y Servicios Múltiples de San Ramón de La Virgen de Sarapiquí, R. L. (Cooproturs R.L.) está conformada en un 55\% por mujeres, quienes demostraron asertividad, liderazgo, compromiso y organización durante el proceso de cuatro años de ejecución de ambos proyectos de extensión. Esto motiva la investigación para ofrecer una sistematización de datos de las experiencias vividas por las mujeres después del proceso de capacitación, con la finalidad última de describir cómo sus percepciones pueden contribuir con el desarrollo de la comunidad. Esta investigación podrá contribuir con la evidencia de las acciones y alcances que tiene la inversión de un proceso de extensión universitaria (2015-2018) y el acompañamiento a las mujeres en los procesos de gestión del turismo, y cómo estos procesos promueven el empoderamiento de las mujeres en un contexto rural.

\section{REVISIÓN DE LITERATURA}

En la IV Conferencia Mundial de las Mujeres en Beijing en el año 1995, se adopta el empoderamiento como una medida positiva para una vida equitativa y segura (Urzelai, 2014). Eskola (2005) propone la Escuela de Empoderamiento de la Mujer, ya que en sus estudios se señaló que la adquisición, el desempeño y el control del poder por parte de las mujeres es un proceso que va en dos direcciones: trabajar en red por los derechos de las mujeres y favorecer las relaciones equitativas entre mujeres y hombres (Baylina, Villarino, Garcia, Mosteiro, Porto y Salamaña, 2019; Pikaza, 2018). El turismo rural es una herramienta de desarrollo que depende de otros factores determinados por el accionar de las mujeres como la planificación y la promoción de los sitios (Rodríguez, Rivera, Idelhadj, Osuna y Rubio 2012), lo cual promueve el empoderamiento y la mejora socioeconómica de la mujer rural en un territorio. Sin embargo, se señala que el control y la gestión del turismo rural es mayoritariamente dominada por hombres. Rodríguez et al. (2012) y Baylina et al. (2019) apuestan por una transformación donde las mujeres se empoderen y logren convertirse en dinamizadoras del desarrollo socioeconómico. 
En el ámbito Latinoamericano, las autoras Erazo, Jiménez y López (2014) sugieren que el empoderamiento se relaciona con el rol de la mujer en lo social, económico, político, legal y ambiental. Por otra parte, resaltan que cuando se trabaja con comunidades, el empoderamiento puede resultar en un cambio transformacional de la mujer, debido a los procesos de autogestión comunitaria. Estos procesos favorecen los planos de acción de la mujer porque se lucha contra el paternalismo y la discriminación por género. Latinoamérica ha avanzado en apoyar a las mujeres en el desarrollo rural, producto de su dinamismo, compromiso y disciplina, demostrado en numerosos roles de la sociedad. Estos roles incluyen la familia, la economía, la política y la cultura. Cabe destacar que, aunque se han validado esfuerzos, sigue persistiendo la inequidad con respecto a derechos e igualdad de oportunidades (Echevarri y Ribero, 2002; Ruiz y Castro, 2011; Soto y Fawaz, 2016). Buendía-Martínez y Carrasco (2013), en su estudio "Mujer, actividad emprendedora y desarrollo rural en América Latina y el Caribe", concluyen que existe una dependencia entre el empoderamiento de la mujer y el desarrollo rural mediante la actividad emprendedora. Así mismo, señalan que las mujeres tienen un papel más protagónico en la economía familiar y en la comunidad rural, con la salvedad de que aún persiste acceso limitado a recursos como los insumos de producción, los servicios, la infraestructura, las organizaciones de apoyo y la tecnología, en comparación con la facilidad que tienen los hombres de gestión de oportunidades para emprender alguna actividad socioeconómica en la ruralidad.

Según el artículo publicado por la Revista Estrategia \& Negocio (2017), Costa Rica ha mostrado cambios significativos en su legislación, que facilitan las oportunidades para la mujer. El mismo reporte indica que las mujeres de Costa Rica son las más emprendedoras de América Latina, liderando el Índice de Mujeres Emprendedoras con 64,7\%, seguido por Perú 64,3\%, Colombia 63,8\% y Chile 62,9\% (E\&N, 2017). Estos datos reflejan que el emprendedurismo es un medio trascendental para la mejora de la calidad de vida de las mujeres, asumiendo que alrededor de una mujer coexiste un entorno familiar, socioeconómico, político y de gestión ambiental. No obstante, las mujeres costarricenses se enfrentan a tres obstáculos cuando se trata de emprender un negocio, argumentan Rodríguez, Leiva y Castrejón (2017), que son el liderazgo, el empoderamiento y los roles en la sociedad. Esto se debe a que la mujer tiene obligaciones implícitas de índole familiar, pero sobre todo social. También, las mujeres sobrellevan un marcado rol de multiplicidad de tareas impuestas por la sociedad y construidas por las costumbres y el patriarcado, así como por los elementos identitarios del colectivo. 


\section{METODOLOGÍA}

El diseño de la investigación será un estudio de caso, el cual es una descripción detallada de un caso en particular y el enfoque será descriptivo (Baxter y Jack, 2008; Flyvbjerg, 2011; Lichtman, 2013). Se escogió el estudio de caso ya que representa un análisis en detalle de un caso con una cantidad extensiva de datos a profundidad. El caso de estudio se centrará en las experiencias de mujeres, las cuáles se capacitaron durante el 2015-2018. El sitio de estudio será San Ramón de La Virgen de Sarapiquí. La población meta de la investigación será de cinco mujeres, las se seleccionarán a través de un muestreo a propósito único con los siguientes criterios: mujeres entre 35 y 65 años de edad, que completaron la capacitación del 2015-2018, que trabajen en procesos de emprendimiento turístico tales como hospedaje, alimentos y bebidas, costura, producción animal y agrícola, diseño y venta de productos locales y artesanías, y líderes comunales que participaron en asociaciones o comités de la comunidad entre el 2015-2018.

La base teórica de esta investigación cualitativa se enfocará en la teoría feminista. Esta teoría se orienta en analizar y visualizar de forma crítica procesos donde las perspectivas feministas sean el centro de atención y donde las experiencias de las mujeres sean la base de la investigación (Grbich, 2007). También busca examinar el rol de las mujeres en la sociedad y como este rol, desde la voz de las mujeres, se percibe y cambia sus vidas en diferentes aspectos sociales (Hatch, 2002). El estudio se enfocará en la investigación cualitativa inductiva ya que las descripciones emergerán de elementos específicos de los datos para convertirse en constructos semánticos generales, conocidos como el contenido o las temáticas (Hatch, 2002). La parte epistemológica de esta investigación se basará en el construccionismo, el cual celebra la creación del conocimiento a través de las experiencias personales e individuales. Toma como premisa que todas las realidades de conocimiento tienen validez y no requieren de comparaciones o hipótesis ya que la realidad es una "construcción humana" (Hatch, 2002, p. 15), y tal cual, es única y legítima para cada individuo. Las percepciones individuales son distintivas y subjetivas ya que están íntimamente relacionadas con las visiones de mundo de cada individuo. Este estudio de caso busca que las mujeres expongan sus experiencias y opiniones del mundo en que viven, y cómo dentro del contexto colectivo de su comunidad ha ido forjado sus percepciones individuales. Las técnicas de recolección de datos triangulados serán entrevistas semiestructuradas, observaciones de campo y recolección de documentos. Las entrevistas serán grabadas y todos los datos serán transcritos. En la investigación 
cualitativa, las entrevistas son instrumentos pertinentes de recolección de datos ya que buscan "entender las experiencias humanas a partir de las perceptivas de quienes las experimentan" (Yegidis y Weinbach, 2006, p. 21). Se utilizará una matriz que indique las preguntas de investigación y de entrevista. Las preguntas de investigación serán abiertas e incluirán preguntas de seguimiento con la finalidad de aclarar y ahondar en las respuestas, así como de explorar las experiencias de los participantes a profundidad. También se llevarán a cabo visitas a la comunidad, donde se utilizará un protocolo de observación de campo. Finalmente, se recogerán diferentes documentos tales como bitácoras, minutas y actas de sesiones, donde existan evidencia de participación de las mujeres en las actividades comunitarias. Como parte de la ética en la investigación cualitativa, las participantes deberán firmar dos consentimientos de acuerdo: uno para el estudio y otro para la entrevista. Se garantizará que toda la información será anónima; las participantes contarán con pseudónimos para asegurar la confiabilidad de la presentación y publicación de los datos. El estudio se basará en el análisis de contenido (Hatch, 2002) y el análisis temático (Braun y Clarke, 2006; Clarke y Braun, 2013).

El análisis de contenido se basa en lecturas constantes, críticas y sistemáticas de los datos. Se crearán códigos que resuman las unidades mínimas semánticas y que determinarán la identificación de categorías temáticas significativas para responder las preguntas de investigación del estudio, las cuáles son: 1. ¿Qué opiniones sobre el proceso de empoderamiento expresan cinco mujeres de San Ramón de La Virgen de Sarapiquí después de haber participado en un proceso de capacitación sobre turismo rural entre el 2015-2018? 2. ¿Cuáles son las percepciones de empoderamiento de cinco mujeres de San Ramón de La Virgen de Sarapiquí después de haber participado en un proceso de capacitación sobre turismo rural entre el 2015-2018? 3. ¿Cuáles son las experiencias de empoderamiento vividas de cinco mujeres de San Ramón de La Virgen de Sarapiquí después de haber participado en un proceso de capacitación sobre turismo rural entre el 2015-2018? El análisis temático se utilizará como método para identificar y analizar los patrones semánticos de esta investigación cualitativa (Braun y Clark, 2006). Se seguirán las siguientes cinco fases recursivas: 
1. Familiarización con los datos: esta fase requerirá una familiarización consciente de los datos de las entrevistas transcritas, los protocolos de observación de campo y la documentación. Se deben llevar a cabo lecturas críticas y sistemáticas de los datos, hasta tener una familiaridad minuciosa con los mismos.

2. Codificación: esta fase se enfocará en una sistematización analítica y un proceso crítico de creación de códigos. Se identificarán "unidades mínimas de significado"meaningful units (Hatch, 2002, p. 163), las cuáles son segmentos específicos que encierran una idea completa y pueden ser una o más palabras, frases u oraciones.

3. Búsqueda de temas: esta fase buscará temas que incluyan los códigos creados. El proceso de identificar y crear temas basado en los códigos será activo e iterativo. Para los temas, hay que tener las preguntas de investigación a mano para la búsqueda objetiva de las respuestas.

4. Revisión de temas: en esta fase se verificará que los temas se ajusten a los códigos y tenga una relación semántica convincente. Algunos temas podrán fusionarse o dividirse, o inclusive descartarse para volver a crear nuevos temas. En esta fase, es importante ser críticos y triangular los temas con colegas externos para dar validez a la creación de los temas.

5. Definición de temas: en esta fase se deberá escribir una descripción detallada de cada tema previamente identificado y creado. Se debe tener claridad del tema y explicar la esencia que encierra con respecto a los códigos creados y la narrativa que se escriba para responder a las preguntas de investigación, con evidencia de ejemplos en los datos.

\section{ANOTACIONES FINALES}

Este estudio de investigación está en curso debido a las implicaciones sociales que se han tenido ante la crisis mundial de salud, provocada por la pandemia del COVID-19. Las investigadoras están en proceso de socializar los alcances de la investigación con la población meta para que conozcan la naturaleza de la investigación y las etapas que se llevarán a cabo. La aplicación y validación de los instrumentos para la recolección de datos ya está en proceso. Como resultados preliminares, se espera aprehender las opiniones, las experiencias vividas y las percepciones de empoderamiento que expresan cinco mujeres de San Ramón de La Virgen de Sarapiquí después de haber participado en un proceso de 
capacitación sobre turismo rural entre el 2015-2018 con el fin de tener una comprensión más consolidada de las experiencias y el rol que juegan las mujeres en la comunidad rural.

Esta investigación contribuirá de forma beneficiosa a la población meta y la comunidad. En primera instancia, servirá como un espacio de reflexión y concientización de las mujeres, haciendo visible el trabajo que hacen para el progreso socio-económico de la comunidad. Dar un espacio y escuchar la voz de las mujeres participantes servirá para comprender sus historias: cómo llegaron donde están, los posibles sacrificios, las vivencias y los caminos que pasaron. Segundo, los resultados de esta investigación también servirán como un primer hito para entender el proceso de empoderamiento y arraigo de las mujeres y el papel que han jugado en la comunidad como pioneras de un proceso. Finalmente, los resultados constituirán un mecanismo sistemático de la ruta que las mujeres participantes han trazado y esto, podrá servir para modelar procesos de aprendizaje y capacitación, y consolidar una guía de retos y experiencias para otras mujeres en otras comunidades.

En términos generales, entender las experiencias de las mujeres, relacionadas con su participación en la dinámica socioeconómica de la comunidad aportará al conocimiento específico en materia de empoderamiento, de comunidad rural y de turismo. Esta investigación contribuirá con la literatura nacional e internacional existente sobre los procesos de empoderamiento de mujeres en contextos rurales.

\section{REFERENCIAS}

Baxter, P., y Jack, S. (2008). Qualitative Case Study Methodology: Study Design and Implementation for Novice Researchers. The Qualitative Report, 13(4), 544-559. Retrieved from https://nsuworks.nova.edu/tqr/vol13/iss $4 / 2$

Baylina, M., Villarino, M., Garcia Ramon, M. D., Mosteiro, M. J., Porto, A. M., Salamaña, I. (2019). Género e innovación en los nuevos procesos de re-ruralización en España. Finisterra, LIV(110), pp. 75-91.

Braun, V., y Clarke, V. (2006). Using thematic analysis in psychology. Qualitative Research in Psychology, 3(2), 77-101. https://doi.org/10.1191/1478088706qp063oa

Clarke, V., y Braun, V. (2013). Teaching thematic analysis: Overcoming challenges and developing strategies for effective learning, The Psychologist 26(2), 120-123.

Buendía-Martínez, I. y Carrasco, I. (2013). Mujer, actividad emprendedora y desarrollo rural en América Latina y el Caribe. Cuadernos de desarrollo rural, 10(72), 21-45.

Erazo, M. I., Jiménez, M. y López, C. (2014). Empoderamiento y liderazgo femenino; su papel en la autogestión comunitaria en el corregimiento El Hormiguero-Valle del Cauca. Avances en Psicología Latinoamericana, 32(1), pp. 149-157. http://dx.doi.org/10.12804/apl32.1.2014.10 
Flyvbjerg, B. (2001). Case Study in Norman K Denzin and Yvonna S. Lincoln, eds., The Sage Handbook of Qualitative Research, 4th Edition (Thousand Oaks, CA: Sage, 2011), Chapter 17, pp. 301-316.

Grbich, C. (2007). Qualitative data analysis: An introduction. City Road, London: Sage Publications.

Hatch, J. A. (2002). Doing qualitative research in education settings. Albany, NY: State University of New York Press.

Litchman, M. (2013). Qualitative Research in Education: A User's Guide. SAGE Publications Inc: Thousand Oaks, United States.

Merriam. S.B. (2009). Qualitative research: A guide to design and implementation. San Francisco, CA: Sage.

Picaza, M. (2018). Espacios propios para las mujeres y procesos de empoderamiento: La Red de Escuelas de Empoderamiento de Bizkaia. Emakunde, Instituto Vasco de la Mujer. C/ Manuel Iradier, 36. 01005 VitoriaGasteiz, pp 323. ISBN: 978-84-09-05159-5. Recuperado de: https://www.emakunde.euskadi.eus/contenidos/informacion/certamen_publicaciones/es_def/adjuntos/v_cert amen_emakunde_2017.pdf

Ruiz, P. Castro, M. (2011). La situación de las mujeres rurales en América Latina. Centro Peruano de Estudios Sociales - CEPES. Primera edición: Lima, julio de 2011, pp 40. ISBN 978-9972-722-18-9. Recuperado de: https://biblio.flacsoandes.edu.ec/libros/digital/55681.pdf

Revista Estrategia \& Negocios (2017, marzo 8). Mujeres ticas son las más emprendedoras de América Latina. Recuperado de: https://www.estrategiaynegocios.net/lasclavesdeldia/1050861-330/mujeres-ticas-son-lasm\%C3\%A1s-emprendedoras-de-am\%C3\%A9rica-latina

Rodríguez, L. Rivera, M. Idelhadj, A. Osuna, M. y Rubio, R. (2012). Mujeres y turismo rural en Andalucía y Norte de Marruecos: una propuesta de investigación sobre emprendimiento femenino, inserción sociolaboral y superación de desigualdades y brecha salarial. Globalización y pluralidad cultural. III Jornada sobre investigación e innovación para la interculturalidad, Universidad de Córdoba, pp 127-150. ISBN: 978-84697-0130-0. Recuperado de: Dialnet-GlobalizacionYPluralidadCultural-560567\%20(2).pdf

Rodríguez, M. Leiva, J. y Castrejón, C. (2017). ¿Cómo son las mujeres que lideran PYMES? Un estudio aplicado en Costa Rica. Revista CEA Vol.3 - No. 5, enero-junio 2017 pp. 29 - 40. ISSN -p 2390-0725, ISSN -e 2422-3182. Recuperado de: https://www.researchgate.net/publication/321037818 Como son las mujeres que lideran PYMES Un e studio aplicado en Costa Rica

Soto, P. y Fawaz, M. (2016). Ser mujer microempresaria en el medio rural. Espacios, experiencias y significados. Cuadernos de Desarrollo Rural, 13(77), 141-165. http://dx.doi.org/10.11144/Javeriana.cdr13-77.smme

Yegidis, B. y Weinbach, R. (2006). Research methods for social workers (5th ed.). New York, NY: Pearson Education Inc. 